\title{
Hemozoin Pigment: An Important Tool for Low Parasitemic Malarial Diagnosis
}

\author{
Sarita Mohapatra',*, Arnab Ghosh', Ruchi Singh'², Dhirendra Pratap Singh'², Bhawna Sharma ${ }^{3}$, \\ Jyotish Chandra Samantaray', Manorama Deb³, Rajni Gaind ${ }^{3}$ \\ ${ }^{1}$ Department of Microbiology, All India Institute of Medical Sciences, New Delhi, India; ${ }^{2}$ National Institute of Pathology, Safdarjung Hospital, New \\ Delhi, India; ${ }^{3}$ Department of Microbiology, Vardhaman Mahavir Medical College and Safdarjung Hospital, New Delhi, India
}

\begin{abstract}
Low parasitemic condition in malaria remains a diagnostic challenge; as the available diagnostic methods failed to detect. Currently, hemozoin $(\mathrm{Hz})$ pigment is gaining attention in the diagnosis of malaria. The major drawback is ease of detection of $\mathrm{Hz}$ in routine practice. A pilot study was conducted to evaluate the role of $\mathrm{Hz}$ pigment and to compare the performance of quantitative buffy coat assay (QBC) and PCR in such conditions. Clinically suspected cases of malaria were examined by both Giemsa stain and immunochromatographic test (ICT). Samples positive by ICT and negative by Giemsa stain were further examined by nested PCR targeting 18S rRNA and QBC for the presence of malaria parasites and pigments. Thirty blood samples fulfilled the inclusion criteria out of which 23 were Plasmodium vivax (Pv), 4 Plasmodium falciparum (Pf), and 3 mixed (Pv and Pf) by immunochromatographic test. Twenty-one out of 30 (70\%) were positive by nested PCR in comparison to $25 / 30$ (83\%) by QBC. Samples containing both malaria parasites and Hz pigment by QBC completely showed concordance with the PCR result. However, $61 \%$ of total samples containing only $\mathrm{Hz}$ pigment were observed positive by PCR. Hz pigment remains an important tool for malaria diagnosis. Identification of leukocytes containing pigments by QBC not only indicates recent malarial infections but also puts light on severity of the disease. QBC assay is a rapid, highly sensitive, and cost-effective method to detect malaria parasites and $\mathrm{Hz}$ pigment especially in low parasitemic conditions.
\end{abstract}

Key words: Plasmodium vivax, Plasmodium falciparum, low parasitemia, hemozoin pigment, QBC, nested PCR

\section{INTRODUCTION}

In malaria, identification of low parasitemic conditions is always a diagnostic challenge. The malaria parasites are maintained as a source of reservoir in these conditions and help in transmission in the community [1]. Correct identification with appropriate treatment of such conditions is highly essential for malaria elimination. It is not only helpful in decreasing the morbidity and mortality but also prevents the transmission. Microscopy of peripheral blood smear and rapid diagnostic tests (RDT) detecting malarial antigen remain the main stay for malaria diagnosis [2]. Although these diagnostic modalities are quite popular in endemic countries, they have their own limitations. Microscopic examination of peripheral blood smears stained with Giemsa needs expertise. In addition, para-

\footnotetext{
- Received 21 October 2015, revised 12 June 2016, accepted 20 July 2016.

*Corresponding author (saritarath2005@yahoo.co.in)

(C) 2016, Korean Society for Parasitology and Tropical Medicine

This is an Open Access article distributed under the terms of the Creative Commons

Attribution Non-Commercial License (http://creativecommons.org/licenses/by-nc/4.0)

which permits unrestricted non-commercial use, distribution, and reproduction in any

medium, provided the original work is properly cited.
}

sitemia below levels of microscopic detection will be falsely diagnosed as negative and continue as potential sources of disease transmission. Similarly, performance level of an RDT can be affected due to varying antigen concentrations in blood and parasitemia levels due to multiple host and parasite factors [3]. Detection of Plasmodium nucleic acid by PCR offers the advantage of high analytical sensitivity in terms of detecting 1 Plasmodium falciparum or 3 Plasmodium vivax parasites per $\mathrm{ml}$ of blood when conventional methods will be negative [4]. However, the usage of PCR requires higher infrastructural support and economic restraints can be a hindrance in its implementation in routine usage in resource-limited settings.

Currently, the role of hemozoin $(\mathrm{Hz})$ pigment in malaria diagnosis is gaining attention [5]. Hz pigment is an important biomarker of malarial infections [6]. These pigments are released during rupture of infected erythrocytes and malaria parasites and phagocytized by the leukocytes during their life cycle. $\mathrm{Hz}$ pigments can be easily detected under a microscope because of their typical morphology. Despite the presence of various sensitive methods like quantitative buffy coat assay (QBC) and PCR for malaria diagnosis, the available literatures 
on $\mathrm{Hz}$ pigment mostly use flow cytometer for its detection on routine basis $[7,8]$. Here, in this study, it was aimed to look for the role of $\mathrm{Hz}$ pigment in the diagnosis of low parasitemic malarial conditions. We also tried to compare the performance of diagnostic methods like QBC and PCR for the detection of $\mathrm{Hz}$ pigment in such conditions.

\section{MATERIALS AND METHODS}

A pilot study was conducted over a period of 3 months. Thirty patients with clinical diagnosis of malaria were included in this study. Ethical clearance was not required for this study as all the samples were routinely sent to the parasitology laboratory of Microbiology Department, and all the tests were performed for all the samples. All the samples were positive by immunochromatographic test (ICT) detecting the LDH antigen and negative by microscopic examination of the peripheral (thin) smear by Giemsa stain. These cases were defined as low parasitemic conditions as no malaria parasites were detected by microscopic examinations after 3 independent observations and positive by ICT (Advantage Mal Card, J Mitra \& Co Pvt. Ltd, New Delhi, India). Blood samples fulfilling the above conditions were further tested by QBC and nested PCR. QBC capillary tubes were examined for presence of malaria parasites and $\mathrm{Hz}$ pigment by all the examiners. All the blood samples were also tested for nested PCR targeting $18 \mathrm{~S}$ rRNA.

\section{DNA extraction}

DNA was extracted from blood ( $50 \mu \mathrm{l})$ using Qiagen DNA isolation kit following manufacturer's instructions $[9,10]$.

\section{Nested PCR based on 18S rRNA amplification}

For nested PCR, the species-specific nucleotide sequences of $18 \mathrm{~S}$ rRNA genes of $P$. falciparum, and $P$. vivax were amplified as

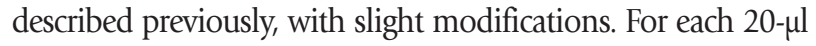
reaction mixture for Nest 1 amplification, $5 \mu \mathrm{l}$ of DNA template (corresponding to approximately 0.25 to $0.5 \mu$ of blood) was added to reaction mixture containing 10 pmoles of each primer (rPLU 1 TCAAAGAATAAGCCATGCAAGTGA and rPLU2 TAACCCTGTTGTTGCCTTAAACTCC), 4 mM MgCl 2 , PCR buffer (50 mM, KCl, 10 mM Tris-HCl), 200 mM of each deoxynucleoside triphosphate, and 1.25 units of Taq DNA polymerase. For Nest 2 assay, $1 \mu$ of Nest 1 PCR product was used with 10 pmoles of respective primers in given PCR conditions. P. vivax VIV F 5'CGCTTCTAGCTTAATCCACATAACTGATAC3'; VIVR 5'ACTTCCAAGCCGAAGCAAAGAAAGTCCTA-3' and $P$. falciparum FAL F 5'TTAAACTGGTTTGGGAAAACCAAATATATT-3'; FAL R ACACAATGAACTCAATCATGACTACCCGTC. The amplified products were visualized in $2 \%$ agarose gels stained with ethidium bromide.

\section{RESULTS}

All the blood samples were positive by ICT. Twenty-three out of 30 samples were detected positive for $P$. vivax, 4 were $P$. falciparum, and 3 were mixed infection showing test bands for both $P$. vivax and $P$. falciparum. All the samples were negative by Giemsa stain examined by 3 independent observers. Twenty-five (83\%) out of 30 samples were found positive by QBC either for malaria parasite or $\mathrm{Hz}$ pigment or both in comparison to $21 / 30(70 \%)$ by nested PCR. Considering PCR as the gold standard for malaria diagnosis, $70 \%$ (21out of 30 samples) were considered as true positive (Table 1).

\section{$\mathrm{QBC}$ result analysis}

QBC assay was performed using fluorescent dye (acridine orange)-coated microcentrifuge capillary tube, where the blood cells are layered as per their specific gravity. The lighter cells remained towards the periphery of the capillary tube where as the heavier cells were positioned towards the cap (Fig. 1). In the buffy coat area, the malarial parasites (with or without $\mathrm{Hz}$ pigments) were found. Leucocytes (e.g. neutrophils, monocytes, and macrophages) containing $\mathrm{Hz}$ pigments were also found in this zone. According to the $\mathrm{QBC}$ observation, our study group was categorized into 3 different subgroups (blood samples containing both malaria parasites and $\mathrm{Hz}$ pig-

Table 1. Detection of malaria parasites and hemozoin $(\mathrm{Hz})$ pigment by quantitative buffy coat (QBC) assay and nested PCR

\begin{tabular}{|c|c|c|c|c|c|}
\hline Groups & PCR positive & PCR negative & $\mathrm{Hz}$ in monocytes & $\mathrm{Hz}$ in macrophages & $\mathrm{Hz}$ in neutrophils \\
\hline Malaria positive and $\mathrm{Hz}$ pigment $(\mathrm{n}=7)$ & 7 & 0 & 7 & 7 & 1 \\
\hline Hz pigment $(n=18)$ & 11 & 7 & 18 & 18 & 5 \\
\hline Negative for malaria and $\mathrm{Hz}$ pigment $(n=5)$ & 3 & 2 & 0 & 0 & 0 \\
\hline Total $(n=30)$ & $21(70 \%)$ & 9 & 25 & 25 & 6 \\
\hline
\end{tabular}




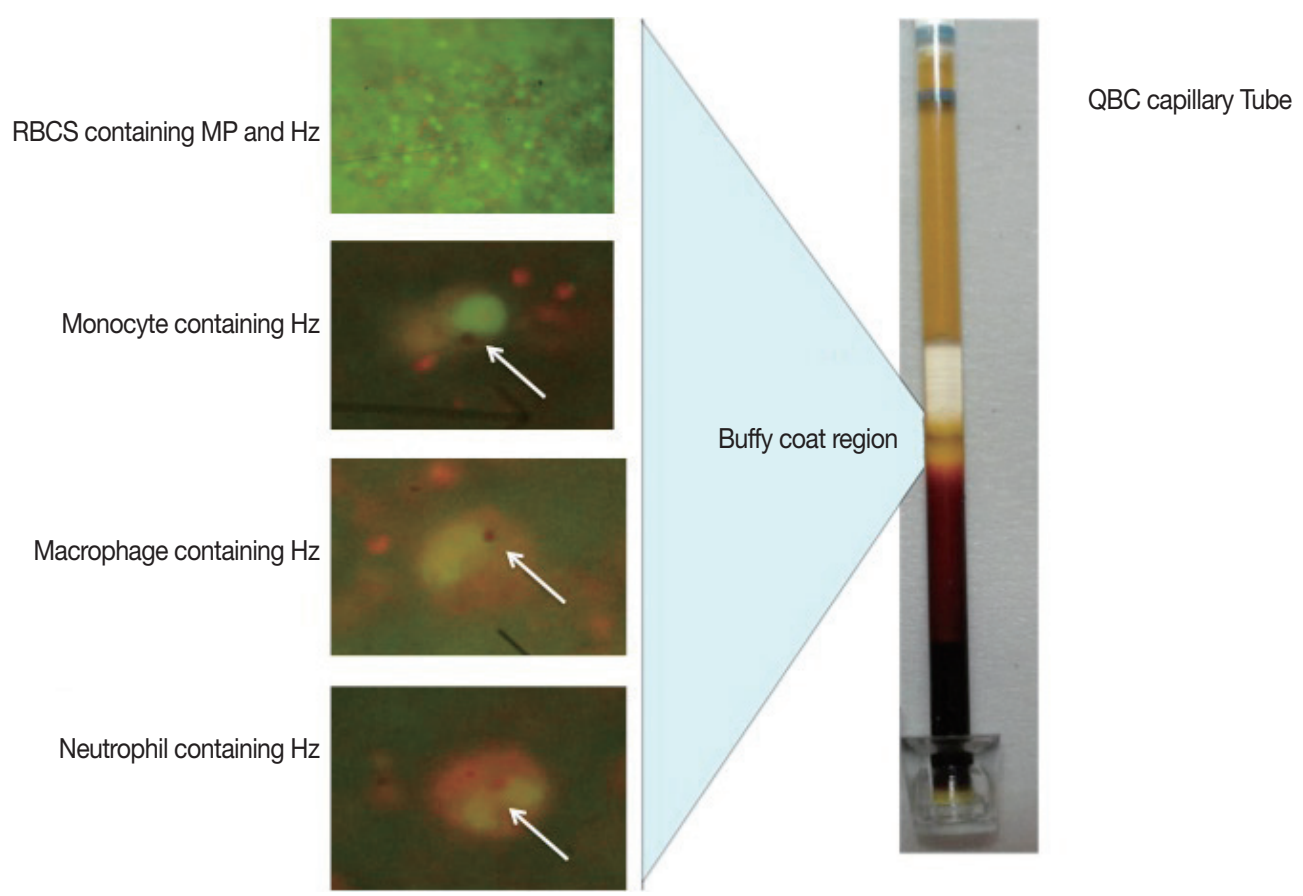

Fig. 1. Quantitative buffy coat (QBC) photograph showing leucocytes containing hemozoin pigment.

Table 2. Correlation of hemozoin $(\mathrm{Hz})$ pigment detection with PCR

\begin{tabular}{lcc} 
& PCR positive & PCR negative \\
\hline Hz positive & 18 & 7 \\
Hz negative & 3 & 2 \\
\hline
\end{tabular}

PCR sensitivity 18/21 (85\%), PCR specificity 2/9 (22\%).

ment, samples showing only $\mathrm{Hz}$ pigment, and samples without malaria parasites and $\mathrm{Hz}$ pigment). QBC was positive in $83 \%$ (25/30) of the total samples. The majority of the samples (18/25) positive by QBC showed only Hz pigment. Seven samples were positive for both malaria parasites and $\mathrm{Hz}$ pigment, and 5 samples were negative for both. All the samples positive for both malaria parasites and $\mathrm{Hz}$ pigment by QBC were found positive by nested PCR. Monocytes and macrophages were the commonest leukocytes containing pigments followed by neutrophils (Table 1).

\section{Nested PCR results analysis}

Twenty-one out of 30 samples (70\%) were found positive by nested PCR. Band size $120 \mathrm{bp}$ was positive for $P$. vivax and 220 bp for $P$. falciparum. All the samples with P. vivax, P. falciparum, and mixed infections were correctly identified by PCR (Fig. 2). All the samples containing malaria parasites and $\mathrm{Hz}$ pigment were positive by PCR in comparison to only $61 \%$ of

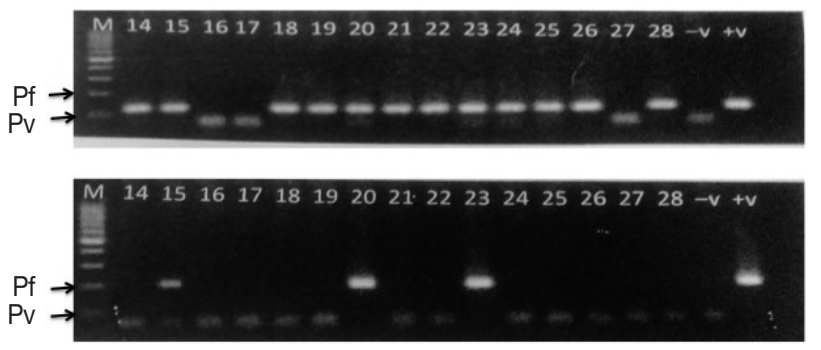

Fig. 2. Gel picture of nested PCR (Pf, 220 bp; Pv, 120 bp).

the samples containing only $\mathrm{Hz}$ pigments positive by PCR.

\section{DISCUSSION}

Low parasitemic condition in malaria remains a diagnostic challenge. Correct diagnosis of such conditions is highly essential as they are the mainstay of malaria transmission in community. Highly sensitive diagnostic tools are required for the detection of such conditions. Hz pigment remains to be the pathognomonic biomarker of malaria pathogenesis [5]. During the intraerythrocytic cycle, Plasmodium synthesizes $\mathrm{Hz}$ pigment by detoxifying the hemoglobin of RBCs. These pigments are formed in all parasitic stages after the early trophozoite stage and persist till the end. During the pathogenesis, the parasites (alone/within the $\mathrm{RBC}$ ) rupture to release $\mathrm{Hz}$ pig- 
ment into the peripheral circulation. Various phagocytic cells such as monocytes, macrophages, and lastly neutrophils engulf the malaria parasites and $\mathrm{Hz}$ pigments. The phagocytic cells are unable to digest these pigments, as they are acid resistant. They can be easily visible under a microscope. They persist within the phagocytic cell till the cells circulate in the blood (life span ranges from 72-296 hr) and finally get deposited within reticuloendothelial cells and various organs [12].

Detection of hemozoin pigment within the phagocytic cell in blood always indicates a recent malarial infection [11]. As a pilot project, we undertook the study to test our hypothesis that detection of hemozoin pigment by QBC of peripheral blood is an alternative diagnostic modality for malaria with low parasitemia by including cases which were diagnosed as positive by RDT but were negative by microscopy of thin smear of peripheral blood. In our study, the majority of samples (defined as low parasitemic) contained only $\mathrm{Hz}$ pigments without presence of any malarial parasite. All the above samples were negative by Giemsa stain and only $70 \%$ of total samples were detected by PCR. Considering PCR as a gold standard for malarial diagnosis, $\mathrm{Hz}$ pigment detection shows a sensitivity of $85 \%$ (18/21) and a specificity of $22 \%$ (2/9). (Table 2) Hence, it proves that $\mathrm{Hz}$ pigment has a certain role in the diagnosis of low parasitemic conditions. The decreased specificity could be as a result of remaining $\mathrm{Hz}$ particle after the treatment.

QBC is a better tool for detection of Hz pigment in comparison to PCR. In the present study, the samples were negative for malarial parasites by QBC. Hence, these samples were unlikely to be positive for malaria by a thick smear since the sensitivity of QBC and thick smears for parasite detection are almost similar. Moreover, the focus of our study was detection of intracellular $\mathrm{Hz}$ pigment in QBC particularly in the lymphocyte-monocyte layer of the buffy coat. Since $\mathrm{Hz}$ pigment is concentrated in the lympho-monolayer in $\mathrm{QBC}$ it is a more sensitive method of pigment detection than the thick smear. Hence, thick smear examination was not done in our study. We also found that the overall positivity of QBC was $83 \%$ in comparison to $70 \%$ by nested PCR. Some of the patients had received antimalarials outside the hospital before the start of investigations. The antimalarials were proved to be inhibitory to PCR. This may be the reason of decreased sensitivity by PCR.

$\mathrm{Hz}$ pigment is a crystalline product having a very typical morphology [12]. It may be linear or non-linear, coarse or fine, and does not require any chemical stain for its detection. Hz-based sensitive tools are highly desirable for the detection of low parasitemic conditions. QBC is a rapid, cost-effective, and highly sensitive tool for $\mathrm{Hz}$ pigment detection [8]. QBC is a much sensitive and specific technique in comparison to others because of 2 reasons. Firstly, the sample is centrifuged in a very high revolution speed so that all the parasites, infected RBCs, and WBCs are concentrated in the buffy coat region. Hence, a very low number of parasites can also be detected and increases its sensitivity. Secondly, the tube is coated with fluorescent dye (acridine orange), which increases the level of detection in comparison to conventional microscopy. It takes less time for sample preparation and interpretation (approximately $30 \mathrm{~min}$ ). In addition to the detection of malaria parasites and $\mathrm{Hz}$ pigment, we can characterize the pigment morphology. Neutrophils containing $\mathrm{Hz}$ pigment have already been proved as a marker for severe malaria and can be easily detected by the QBC assay as shown in Fig. 1. In the present study, we found that 6 out of 30 samples were positive for neutrophils containing $\mathrm{Hz}$ pigment, and 3 out of 5 were having various complications. Samples with neutrophils containing pigment can be alerted from the beginning, and adequate care can be taken to reduce the morbidity and mortality. Other advantages using QBC assay include semi-quantitative detection of packed cell volume or hemoglobin level. This assay can also detect other hemoparasites (such as Babesia, microfilaria, trypanosome, and Donovan bodies of Leishmania donovani).

Molecular methods are proven to have highest sensitivity and specificity among all the diagnostic modalities available for malaria. However, in the current study, few cases were found positive by ICT and QBC but negative by PCR. In a retrospective data analysis, many of these patients were found to receive 1 or 2 doses of antimalarials prior to the sampling. Moreover, few samples exhibited only $\mathrm{Hz}$ pigments in QBC, and positive by both ICT and PCR. Hence, the detection of $\mathrm{Hz}$ pigments in low parasitemic conditions can be an adjunct for malarial diagnosis.

The limitation of this study was that we did not examine the role of $\mathrm{Hz}$ pigment detection by $\mathrm{QBC}$ in samples which were both negative by microscopy and RDTs. This was a pilot study by which we wanted to validate the utility of $\mathrm{QBC}$ for $\mathrm{Hz}$ detection for which we needed a substantial number of samples, which were negative for parasitic forms by microscopy. The majority of such samples received during the study period were RDT positive, and hence we used such samples to see 
whether $\mathrm{Hz}$ detection by QBC serves any purpose.

Low parasitemic conditions in malaria need to be evaluated carefully. Hz pigment is a very good marker for malarial infection. Its use in the detection of low parasitemic condition will be highly useful. It not only signifies the recent malarial infection but also puts light on severity of the disease. In the present study, we found that QBC is a rapid, highly sensitive, and cost-effective method to detect $\mathrm{Hz}$ pigment in comparison to PCR in low parasitemic conditions.

\section{CONFLICT OF INTEREST}

The authors declare that they have no competing interest.

\section{REFERENCES}

1. Shekaleghe S, Alifrangis M, Mwanziva C, Enevold A, Mwakalinga S, Mkail H, Kavishe R, Manjurano A, Sauerwein R, Drakeley C, Bousema T. Low density parasitaemia, red blood cell polymorphisms, and Plasmodium falciparum specific immune responses in low endemic areas of northern Tanzania. BMC Infect Dis 2009; 9: 69.

2. Harris I, Sharrock WW, Bain LM, Gray KA, Bobogare A, Boaz L, Lilley K, Krause D, Vallely A, Johnson ML, Gatton ML, Shanks GD, Cheng Q. A large proportion of asymptomatic Plasmodium infections with low and sub-microscopic parasite densities in the low transmission setting of Temotu province, Solomon Islands; challenges for malaria diagnostics in elimination setting. Malar J 2010; 9: 254.

3. Francisca L, Kusnanto JH, Satoto TB, Sebayang B, Supriyanto, Andriyan E, Bangs MJ. Comparison of rapid diagnostic test Plasmotec Malaria-3, microscopy, and quantitative real-time PCR for diagnoses of Plasmodium falciparum and Plasmodium vivax infections in Mimika Regency, Papua, Indonesia. Malar J 2015; 14: 103.

4. Morassin B, Fabre R, Berry A, Magnaval JF. One year's experience with the polymerase chain reaction as a routine method for the diagnosis of imported malaria. Am J Trop Med Hyg 2003; 66: 503-508.

5. Hänscheid T, Egan TJ, Grobusch MP: Hemozoin: from melatonin pigment to drug target, diagnostic tool, and immune modulator. Lancet Infect Dis 2007; 7: 675-685.

6. Delahunt C, Horning MP, Wilson BK, Proctor JL, Hegg MC. Limitation of haemozoin-based diagnosis of Plasmodium falciparum using dark field microscopy. Malar J 2014; 13: 147.

7. Frita R, Rebelo M, Pamlona A, Vigario AM, Mota MM, Grobusch MP Hanscheid T. Simple flow-cytometric detection of haemozoin containing leukocytes for research on diagnosis, immunology and drug sensitivity testing. Malar J 2011; 10: 74.

8. Mohapatra S, Samantaray JC, Arulselvi S, Panda J, Dang N, Saxena R. Comparative evaluation of two flow cytometric analysers as diagnostic tools for the automated detection of malaria. Ann Clin Lab Sci 2014; 44; 82-86.

9. Singh B, Bobogare A, Cox-Singh J, Snounou G, Abdullah MS, Rahman HA. A genus- and species-specific nested polymerase chain reaction malaria detection assay for epidemiologic studies. Am J Trop Med Hyg 1999; 60: 687-692.

10. Valecha N, Pinto RG, Turner GD, Kumar A, Rodrigues S, Dubhashi NG, Rodrigues E, Banaulikar SS, Singh R, Dash AP, Baird JK. Histopathology of fatal respiratory distress caused by Plasmodium vivax malaria. Am J Trop Med Hyg 2009; 81: 758-762.

11. Day NP, Pham TD, Phan T, Dinh XS, Pham PL, Ly VC, Tran TH, Nguyen TH, Bethell DB, Nguyan HP, Tran TH, White NJ. Clearance kinetics of parasites and pigment-containing leukocytes in severe malaria. Blood 1996; 88: 4694-4700.

12. Baura M, Frita R, Gois A, Carvalho T, Hancsheid T. The haemozoin conundrum: is malaria pigment immune-activating, inhibiting, or simply a bystander? Trends Parasitol 2013; 29: 469-476. 